Rev. Elet. em Gestão, Educação e Tecnologia Ambiental (e-ISSN: 2236-1170)

\title{
ANÁLISE DA RELAÇÃO ENTRE A PRODUÇÃO E O CONSUMO DE ÁGUA NA GRANDE GOIÂNIA UTILIZANDO O MÉTODO ESTATÍSTICO DE PREVISÃO DE HOLT-WINTERS
}

\author{
Victor Freitas de Azeredo Barros ${ }^{1}$, José Elmo de Menezes ${ }^{2}$ \\ ${ }^{1}$ Instituto Federal de Educação, Ciência e Tecnologia de Goiás \\ vfbarros@gmail.com, \\ ${ }^{2}$ Professor no Instituto Federal de Goiás, Campus Inhumas e Pontifícia Universidade Católica de Goiás \\ jelmo@ucg.br
}

\begin{abstract}
All in planet Earth, the volume of salt water found in oceans and seas is about $97 \%$; the other $3 \%$ is fresh, but they are found in glaciers or underneath the earth surface, in aquifers, and it is difficult to access them. Only $0.007 \%$ of freshwater is found in rivers, lakes and in the atmosphere - the easy access water to human being. Brazil has $13.7 \%$ of surface freshwater in the World and $70 \%$ of this amount is at Amazon. The other $30 \%$ are distributed throughout the country. The growing of water needed by the population concentration and the water disponibility in certain areas demands to plan how to use this water. Based on these points some statistical methods were used as models of regression and timing series, taking Holt-Winters Methodology as a basic tool to model the water consumption withing coming decades.
\end{abstract}

Index Terms: Holt-Winters, Statistical Model, Timing Series, Water Consumption.

\section{RESUMO}

Sabe-se que 97,5\% da água disponível na terra é salgada e se encontram em oceanos e mares. Já a água doce, 2,493\% se encontra em geleiras ou regiões subterrâneas (aquíferos), de difícil acesso restando apenas $0,007 \%$ de fácil acesso para o consumo humano, encontradas em rios, lagos e na atmosfera. Desta pequena parcela, o Brasil detém 13,7\%, sendo $70 \%$ localizados na Região Amazônica e os $30 \%$ restantes distribuídos desigualmente pelo país. A região do centro oeste detém de $15,7 \%$ destes recursos. Com base nos dados, há uma necessidade de planejamento do uso da água decorrentes da crescente demanda devido ao crescimento populacional, infraestrutura e sua disponibilidade na natureza. Baseados nessas premissas propõem-se uma modelagem da produção de água na Grande Goiânia para as próximas décadas com uma das principais fontes de abastecimento de água da região - a Barragem do João Leite - utilizando métodos estatísticos como séries temporais dando uma atenção especial ao método de HoltWinters

Palavras Chaves: Holt Winters, Modelagem Estatística; Séries Temporais; Consumo de Água 


\section{INTRODUÇÃO}

Quando se pensa na distribuição de água no planeta, sabe-se que $97 \%$ desta água se encontram nos oceanos e mares, restando apenas 3\% de água doce. Desta pequena parcela de água doce, 2,493\% se encontram em geleiras e aquíferos, sendo de difícil acesso para o consumo humano, e 0,007\% é de fácil acesso, encontradas em rios, lagos e na atmosfera (REDE DAS ÁGUAS, 2001).

O Brasil é o país mais rico do mundo em recursos hídricos, com $13,7 \%$ de toda a água doce disponível no planeta, e desse total, 70\% estão localizados na Região Amazônica - a maior área úmida do mundo - e os $30 \%$ restantes se distribuem desigualmente pelo país, atendendo 95\% da população (VIEIRA, 2006).

A cidade de Goiânia, Capital do Estado de Goiás, possui uma área de $739 \mathrm{~km}^{2}$ e uma população estimada em 1.265.394 habitantes. Ao longo dos anos, tanto a Capital como o seu entorno, se encontram em plena expansão econômica, com crescimento de seus polos industriais, agricultura e, principalmente, a agroindústria (SEPIN, 2007). Com essa expansão demográfica, ocorrendo de forma significativa, vários problemas de infraestrutura ocorrem, como o alto consumo de água e graves problemas de saneamento básico.

A topografia da cidade de Goiânia e seu entorno é composta por 22 sub-bacias hidrográficas que deságuam nos Ribeirões João Leite, que corresponde com $52 \%$ do suprimento de água da Capital, e o Rio meia Ponte, correspondendo com $48 \%$ do suprimento de água da Capital (SANEAGO, 2010).

A bacia do Ribeirão João Leite, localizada no Centro-Sul Goiano possui uma área de $767 \mathrm{~km}^{2}$ e coincide com a área parcial de sete municípios goianos: Anápolis, Goianápolis, Campo Lindo de Goiás, Goiânia, Nerópolis, Ouro Verde de Goiás e Teresópolis de Goiás (RABELO, 2009). A Figura 1 indica o seu posicionamento geográfico.

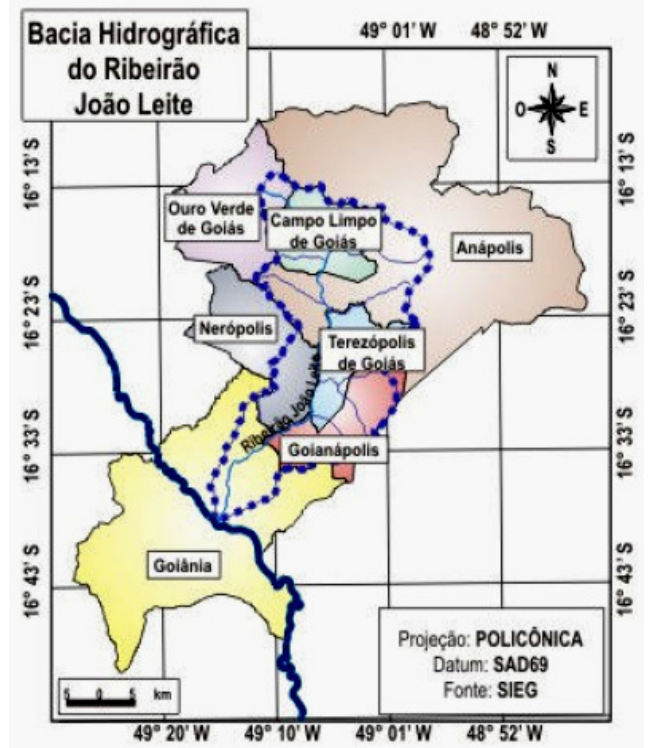

Figura. 1. Localização da bacia hidrográfica do Ribeirão João Leite em Goiás. Fonte: Sistema Estadual de Estatística e de Informações Geográficas de Goiás (SIEG). Disponível em: www.sieg.go.gov.br. 


\section{Rev. Elet. em Gestão, Educação e Tecnologia Ambiental (e-ISSN: 2236-1170)}

Devido ao crescimento da cidade de Goiânia e seu entorno e, consequentemente, um aumento do consumo de água, surge à necessidade de uma nova barragem para o abastecimento de água. Surge a barragem João Leite.

Hoje Goiânia conta com dois sistemas de água tratada: sistema João Leite e o sistema Meia Ponte. Cada um contribui com $02 \mathrm{~m}^{3} / \mathrm{s}$ para atender Goiânia. A barragem é parte do sistema produtor do João Leite que é composto ainda pelo reservatório, uma estação elevatória de água bruta, uma estação de tratamento de água e uma estação de tratamento de água tratada. Com a conclusão da barragem, explica o diretor de engenharia da SANEAGO, Mário João de Souza, serão construídas as elevatórias, adutoras de água bruta e estação de tratamento e adutora de água tratada (SANEAGO, 2010).

Segundo a SANEAGO - Saneamento de Goiás S/A, Goiânia/GO - a barragem João Leite abastece a Grande Goiânia - constituída pela capital Goiânia e partes de municípios vizinhos - tem capacidade total para $148.792 .243 \mathrm{~m}^{3}$ ao ano.

Mediante das informações acima, fazemos o seguinte questionamento: O volume de água da barragem João Leite que atende a Grande Goiânia é suficiente? Em caso afirmativo, até quando?

Sabe-se que há uma necessidade de um estudo voltado ao consumo de água, pois devido a demanda requerida pela concentração populacional em Goiânia e região, tem-se a necessidade de um planejamento do uso de água decorrente entre a crescente demanda requerida pela concentração da população, sua disponibilidade na natureza e infraestrutura.

Diante destas premissas, verificou a produção de água da Barragem do João Leite e, a partir deste levantamento, fizemos uso de métodos de previsão como ferramentas para prever a quantidade de produção anual da Barragem do João Leite e o consumo médio de água para os próximos anos. Com isso, foi estabelecido até quando o abastecimento de água estará em uma condição confortável com respeito ao abastecimento de água e suportará o alto consumo da região da Grande Goiânia.

Segundo Makridakis, Wheelwright \& Hyndman (1998), sabe-se que a previsão, como ferramenta, deve ser executada considerando alguns importantes passos:

(1) Definição do Problema: Conhecer o problema; utilidade da previsão; entre outros;

(2) Coleta de Informações: Busca e coleta de dados;

(3) Análise Preliminar dos Dados: Através de gráficos e medidas descritivas;

(4) Definição do Modelo: Escolha e ajuste de modelos; e

(5) Uso do Modelo: Uso e avaliação do modelo de previsão (MAKRIDAKIS, WHEELWRIGHT, HYNDMAN, 1998).

Tendo esses componentes e admitindo a existência de algum padrão geral e não aleatório de comportamento, ou seja, crescimento ou decrescimento claramente definidos, elas poderão ser descritas por funções matemáticas, com as quais será possível fazer previsões futuras de comportamento do fenômeno estudado naquele instante.

Baseado nestas premissas utilizou-se de métodos estatísticos, como modelos de regressão e séries temporais, com uma atenção especial no método de Holt-Winters ${ }^{1}$, como ferramentas para modelagem da produção de água na Grande Goiânia a partir da Barragem do João Leite para os próximos anos.

\footnotetext{
${ }^{1}$ Método proposto por Holt (1957) e Winters (1960). É baseado em três equações de suavização: nível, tendência e sazonalidade e, dependendo da sazonalidade, pode ser modelado de forma multiplicativa ou aditiva.
} 
Rev. Elet. em Gestão, Educação e Tecnologia Ambiental (e-ISSN: 2236-1170)

O conhecimento desses efeitos é necessário para auxiliar no planejamento de abastecimento de água tratada na região como também para fornecer ferramentas estatísticas na detecção e solução de modelos de séries temporais.

\section{METODOLOGIA}

Inicialmente, fez-se um levantamento bibliográfico em periódicos científicos, dissertações de mestrado e teses de doutorado dos principais programas de pós-graduação do Brasil e exterior.

Paralelamente a pesquisa bibliográfica fez-se uma busca de dados - obtidos pelo órgão de saneamento do estado, a SANEAGO - e a partir destes dados formou-se um banco de dados com informações sobre a produção de água na Grande Goiânia e a população beneficiada dessa produção da Barragem do João Leite indexada anualmente, conforme pode ser visto na Tabela 1. Vale ressaltar que nem toda água consumida por estas cidades que fazem partem deste complexo são de uma única barragem.

Tabela 1. População Beneficiada na Grande Goiânia e a Produção de Água na Barragem do João Leite indexada anualmente de 1975 a 2006 Fonte: SANEAGO, 2010.

\begin{tabular}{|c|c|c|c|c|}
\hline ANO & POPULAÇÃO (hab.) & $\begin{array}{c}\text { POPULAÇÃO ATENDIDA } \\
(\%)\end{array}$ & $\begin{array}{l}\text { POPULAÇÃO ATENDIDA } \\
\text { (hab.) }\end{array}$ & $\begin{array}{l}\text { PRODUZIDO } \\
\left(\mathrm{m}^{3} / \text { ano }\right)\end{array}$ \\
\hline 1975 & 711.798 & 30,917 & 220.065 & 25.332 .701 \\
\hline 1976 & 725.515 & 34,487 & 250.207 & 27.954 .466 \\
\hline 1977 & 775.436 & 38,347 & 297.357 & 36.253 .884 \\
\hline 1978 & 828.792 & 40,657 & 336.959 & 40.689 .160 \\
\hline 1979 & 885.819 & 41,910 & 371.249 & 42.956 .633 \\
\hline 1980 & 943.681 & 43,253 & 408.168 & 48.014 .862 \\
\hline 1981 & 971.236 & 46,606 & 452.650 & 50.456 .074 \\
\hline 1982 & 999.597 & 48,922 & 489.021 & 54.316 .290 \\
\hline 1983 & 1.028 .785 & 54,195 & 557.554 & 55.975 .249 \\
\hline 1984 & 1.058 .826 & 57,428 & 608.065 & 59.848 .439 \\
\hline 1985 & 1.089 .743 & 58,962 & 642.529 & 64.902 .743 \\
\hline 1986 & 1.121 .564 & 59,758 & 670.221 & 75.460 .501 \\
\hline 1987 & 1.154 .314 & 63,906 & 737.675 & 77.761 .550 \\
\hline 1988 & 1.187 .904 & 59,106 & 702.121 & 77.445 .074 \\
\hline 1989 & 1.222 .472 & 72,411 & 885.208 & 72.010 .750 \\
\hline 1990 & 1.258 .047 & 76,507 & 962.488 & 94.038 .342 \\
\hline 1991 & 1.294 .486 & 78,458 & 1.015 .632 & 101.371 .288 \\
\hline 1992 & 1.327 .295 & 79,807 & 1.059 .277 & 96.139 .545 \\
\hline 1993 & 1.360 .916 & 82,245 & 1.119 .291 & 95.047 .102 \\
\hline 1994 & 1.395 .402 & 83,847 & 1.169.999 & 98.934 .083 \\
\hline 1995 & 1.430 .759 & 83,483 & 1.194 .446 & 107.774 .083 \\
\hline 1996 & 1.466.229 & 81,464 & 1.194 .446 & 105.827 .683 \\
\hline 1997 & 1.493 .553 & 82,043 & 1.225 .355 & 111.378 .082 \\
\hline 1998 & 1.433 .718 & 89,764 & 1.286 .962 & 115.595 .717 \\
\hline 1999 & 1.501 .820 & 89,345 & 1.341 .807 & 116.143 .695 \\
\hline 2000 & 1.573 .158 & 88,283 & 1.388 .835 & 115.345 .587 \\
\hline 2001 & 1.459 .283 & 93,757 & 1.368 .180 & 114.702 .002 \\
\hline
\end{tabular}


Rev. Elet. em Gestão, Educação e Tecnologia Ambiental (e-ISSN: 2236-1170)

\begin{tabular}{lllll}
\hline $\mathbf{2 0 0 2}$ & 1.631 .553 & 87,703 & 1.430 .928 & 113.702 .836 \\
$\mathbf{2 0 0 3}$ & 1.666 .630 & 88,484 & 1.474 .697 & 113.105 .263 \\
$\mathbf{2 0 0 4}$ & 1.702 .462 & 88,075 & 1.499 .450 & 116.765 .309 \\
$\mathbf{2 0 0 5}$ & 1.739 .063 & 88,027 & 1.530 .843 & 123.972 .339 \\
$\mathbf{2 0 0 6}$ & 1.776 .452 & 88,880 & 1.578 .906 & 127.694 .395 \\
\hline
\end{tabular}

Tendo em mãos este conjunto de dados, fez-se uma análise estatística dos dados verificando na literatura qual o melhor método e modelo de previsão para esta série de dados observando parâmetros de ajustes de precisão para serem calculados (tempo, crescimento da população, etc). Fez-se também o tratamento dos dados, utilizando o modelo de alisamento exponencial que é bastante utilizada para suavização e previsão de uma série temporal.

Pelas análises preliminares feitas sobre os dados, revelou-se a presença de componentes de tendência e de sazonalidade, tornando-se apropriado a utilização do método de Holt-Winters para efetuar tais previsões. A tendência se deve ao fato de que a população vem crescendo ao longo dos anos e, portanto o consumo de água também. A sazonalidade deve ocorrer pelo fato que nos meses mais quentes ou mais secos o consumo de água também aumenta e provavelmente diminuem nos meses chuvosos e mais frios.

Os modelos de Holt-Winters descrevem apropriadamente dados em que se verifica a ocorrência de tendência linear, além de componente de sazonalidade (PELLEGRINI; FLOGLIATTO, 2000). Sua aplicação é válida pra séries não estacionárias.

Morettin \& Toloi (2004) comentam que as vantagens desse modelo são: fácil entendimento, aplicação não dispendiosa, adequada para série com padrão de comportamento mais geral. E que as desvantagens são: dificuldades de determinar os valores mais apropriados das constantes de suavização e/ou impossibilidade de estudar as propriedades estatísticas, tais como média e variância da previsão e, consequentemente, a construção de um intervalo de confiança.

O modelo de Holt-Winters é baseado em três equações alisadoras. Uma para o nível, outra para tendência e outra para sazonalidade. A sazonalidade pode ter efeito multiplicativo ou aditivo que se diferem por:

Holt-Winters Aditivo. É utilizado quando a amplitude da variação sazonal mantém-se constante, ou seja, a diferença entre o maior e o menor ponto de demanda nos ciclos permanece constante com o passar do tempo.

Holt-Winters Multiplicativo. É utilizado quando a amplitude da variação sazonal aumenta com o tempo, ou seja, a diferença entre o maior e o menor ponto de demanda nos ciclos cresce com o passar do tempo (MORETTIN; TOLOI, 2004).

As equações básicas para realizar previsões dos principais métodos de suavização exponencial, podem ser modelados de forma multiplicativa ou aditiva (HYNDMAN; KHANDAKAR, 2008). A Tabela 2 abaixo mostra as equações comparativas dos modelos Multiplicativo e Aditivo de Holt-Winters. 
Rev. Elet. em Gestão, Educação e Tecnologia Ambiental (e-ISSN: 2236-1170)

Tabela 2. Equações comparativas dos modelos de Holt-Winters multiplicativo e aditivo quanto a Nível, Tendência, Sazonalidade e Previsão.

\begin{tabular}{|c|c|c|}
\hline & Holt-Winters Multiplicativo & Holt-Winters Aditivo \\
\hline & $(1)$ & $(5)$ \\
\hline Nível & $L_{t}=\alpha \frac{y_{t}}{s_{t-1}}+(1-a)\left(L_{t-1}+b_{t-1}\right)$ & $L_{t}=a\left(Y_{t-} S_{t-\Omega}\right)+(1-a)\left(L_{t-1}+b_{t-1}\right)$ \\
\hline Tendência & $b_{t}=\beta\left(L_{t}-L_{t-1}^{(2)}\right)+(1-\beta) b_{t-1}$ & $b_{t}=\beta\left(L_{t}-L_{t-1}^{(6)}\right)+(1-\beta) b_{t-1}$ \\
\hline Sazonalidade & $s_{t}=\gamma \frac{Y_{t}}{L_{t}}+(1-\gamma) 5_{t-s}$ & $S_{t}=\gamma\left(Y_{t}-L_{t}\right)+(1-y) S_{t-s}$ \\
\hline Previsão & $\begin{array}{c}(4) \\
F_{\mathrm{i}+\mathrm{m}}=\left(L_{\mathrm{i}}+b_{\mathrm{i}-\mathrm{m}}\right) s_{\mathrm{i}-\mathrm{s}+\mathrm{m}}\end{array}$ & $\begin{array}{c}(8) \\
F_{t+m}=L_{i}+b_{i-m}+s_{i-1+m}\end{array}$ \\
\hline
\end{tabular}

Ressalta-se que:

- $s$-comprimento da sazonalidade;

- $\quad L_{t}-$ nível da série;

- $b_{t}$ - tendência;

- $S_{t}$ - componente sazonal;

- $\quad F_{t+m}$ - previsão para o período m adiante;

- $Y_{t}$-valor observado; e

- $\quad \alpha, b, \gamma$-parâmetros exponenciais alisadores, do nível, da tendência e da sazonalidade, respectivamente.

Criou-se então um gráfico que mostra visualmente, o aumento da produção de água na Grande Goiânia utilizando o método de Holt-Winters e posteriormente, depois de toda análise estatística, criou-se um novo banco de dados com a previsão da produção de água na Grande Goiânia e a população beneficiada desta produção da Barragem do João Leite, indexada anualmente. A partir deste banco de dados verificou se a Barragem do João Leite suportará, e até quando ela suportará o consumo de água nos próximos anos da Grande Goiânia.

O método utilizado neste trabalho, desenvolvido por Box \& Jenkins (1976), baseia-se na ideia que a série temporal pode ser parcialmente explicada por ela mesma, através de parâmetros auto regressivos que podem explicar situações anteriores. Neste caso, utilizamos este método para estimar a demanda de água em Goiânia porque a água depende de muitos fatores relacionados ao tempo e também por ser um componente sazonal, ou seja, aumento de consumo no verão, aumento da população, infraestrutura, etc.

Ehlers (2009) também faz referência a esta mesma análise em seu livro, utilizando um banco de dados contendo os números mensais de mortes por doenças de pulmão (bronquite, enfisema pulmonar e asma) no Reino Unido entre Janeiro de 1974 e dezembro de 1979.

A análise de séries temporais é uma área de pesquisa relevante em diversos campos do conhecimento, tendo como principal objetivo em suas pesquisas, providenciar uma previsão, quando o modelo matemático de um fenômeno é desconhecido ou incompleto. Uma série temporal consiste de medidas ou observações previamente obtidas de um fenômeno que são realizadas sequencialmente sob um intervalo de tempo (SAMOHYL; RUBSON; MATTOS, 2001). Se estas observações consecutivas são dependentes uma da outras então é possível conseguir-se uma previsão. 
Como o volume de dados é muito grande, o uso de uma ferramenta computacional foi necessário e, para isto, utilizou-se o Programa Estatístico $R^{2}$. O programa $R$ é um software gratuito e de software aberto que propicia excelente ambiente para análise estatística de alta qualidade.

\section{RESULTADOS E DISCUSSÃO}

Após todo o estudo em teses, artigos e outras fontes bibliográficas, foi feito uma coleta de dados da produção de água da Barragem do João Leite de 1975 a 2006 como também a população atendida, conforme foi observado na tabela anterior (Tabela 1). Estes dados foram obtidos pelo órgão de saneamento do estado, a SANEAGO. Vale ressaltar que, como nem toda a água consumida pelas cidades que formam a Grande Goiânia são a partir de uma única barragem, foi feita uma estimativa para obter apenas os dados referentes à produção de água na Barragem do João Leite para a Grande Goiânia. A tendência de aumento da produção de água da Barragem do João Leite é facilmente visualizada na Figura 2.

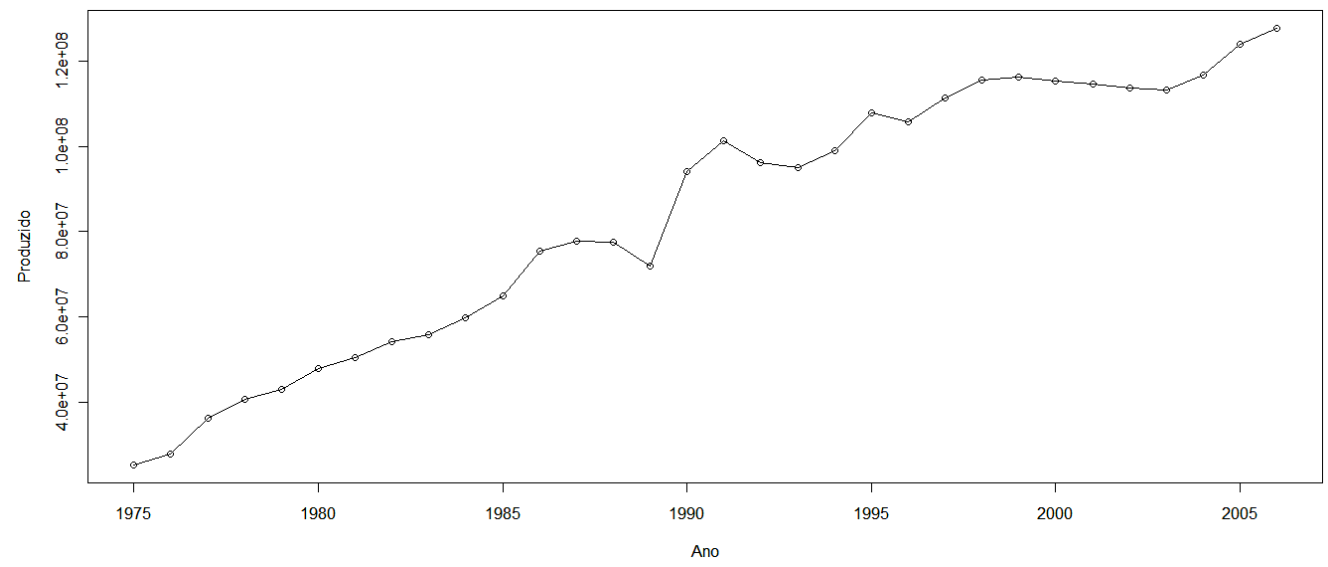

Figura 2. Produção de água da barragem do João Leite nos anos de 1975 a 2006.

Como a série observada acima apresenta níveis de sazonalidade e tendência, um dos métodos mais utilizados é o Holt-Winters. Assim, foi utilizada a função HoltWinters do programa estatístico R para fazer a estimação dos parâmetros de suavização e o estado inicial determinados pela função de verossimilhança bem como o critério de seleção realizado pelo AIC da produção de água da Barragem do João Leite. A Tabela 3 mostra os estados iniciais para as equações de recorrência, as constantes de suavização $(\alpha, \beta$ e $\gamma)$, determinadas a partir da soma dos quadrados dos erros de previsão e os critérios de informação.

\footnotetext{
${ }^{2}$ R DEVELOPMENT CORE TEAM. R: A language and environment for statistical computing. R Foundation for Statistical Computing, Vienna, Austria. ISBN 3-900051-07-0, URL http://www.R-project.org/. 2011.
} 
Rev. Elet. em Gestão, Educação e Tecnologia Ambiental (e-ISSN: 2236-1170)

Tabela 3. Valores iniciais para as equações de recorrência e parâmetros de suavização do modelo de Holt-Winters.

\begin{tabular}{c|c|c|c|c|c}
\hline \multicolumn{2}{c|}{ Valores Iniciais } & \multicolumn{2}{|c|}{ Parâmetros de Suavização } & \multicolumn{2}{c}{ Critérios de Informação } \\
\hline$l_{o}$ & 23131172.0787 & $\alpha$ & 0.002 & AIC & 1093.673 \\
$b_{0}$ & 1.1676 & $\beta$ & 0.002 & AICc & 1095.980 \\
$s_{0}$ & 0.0519 & $\gamma$ & 0.9216 & BIC & 1101.001 \\
\hline
\end{tabular}

A Figura 3 ilustra o histograma e QQ-Plot dos resíduos para o modelo de Holt-Winters, que quando submetidos ao teste de Shapiro-Wilk para normalidade apresentaram como resultado $\mathrm{W}=0,9873$ e $p$-value $=0,9632$, comprovando que se aproximam suficientemente da distribuição normal. O teste Jarque-Bera com $X^{2}=0,2319$ e $p$-valor $=0,8905$ também demonstrou que 0 terceiro e quarto momentos da distribuição empírica coincidem com os da normal, ao nível de significância de $5 \%$. Os resultados dos testes de Shapiro-Wilk e o teste de Jarque-Bera podem ser visualizados na Tabela 4.

(a)

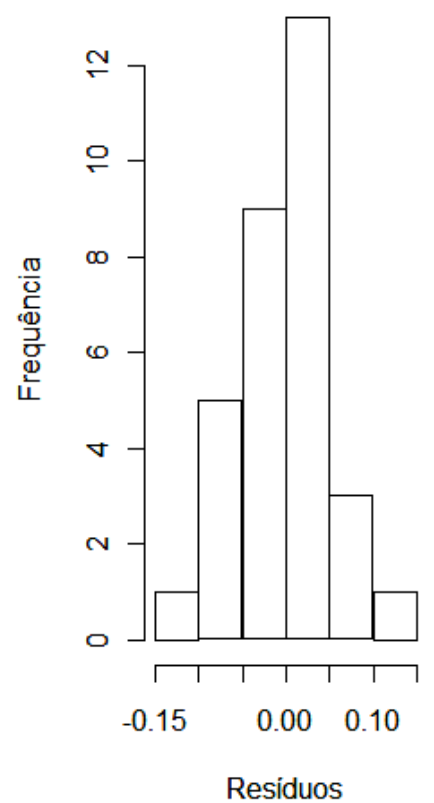

(b)

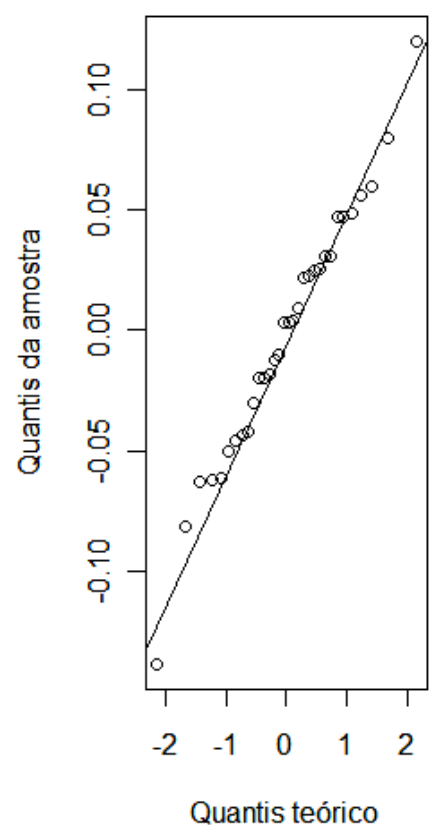

Figura 3. Histograma e QQ-Plot dos resíduos para o modelo de Holt-Winters.

Tabela 4. Testes de normalidades Shapiro-Wilk e Jarque-Bera.

\begin{tabular}{ccc}
\hline & Teste de Normalidade & \\
& Shapiro-Wilk & Jarque-Bera \\
\hline Estatística & $\mathrm{W}=0,9873$ & $\mathrm{X}^{2}=0,2319$ \\
- value & 0,9632 & 0,8905
\end{tabular}

Como os modelos de Holt-Winters foram validados e estão aptos para realizar previsões, os mesmos foram descritos após a determinação dos parâmetros através das fórmulas das quais já foram representadas (Tabela 2 ). 
Após esta análise foi gerado pelo programa estatístico $R$ a produção estimada de 2006 a 2025, gerando um novo banco de dados conforme Tabela 5. A previsão da produção de água na Grande Goiânia e a população beneficiada desta produção da Barragem do João Leite pode ser facilmente visualizada na Figura 4 (com o modelo aditivo de Holt-Winters) e Figura 5 (com o modelo multiplicativo de Holt-Winters).

Tabela 5. Previsão da População Beneficiada na Grande Goiânia e a Produção de Água na Barragem do João Leite indexada anualmente de 2007 a 2025.

\begin{tabular}{|c|c|c|c|c|}
\hline ANO & $\begin{array}{l}\text { POPULAÇÃO } \\
\text { (hab.) }\end{array}$ & $\begin{array}{c}\text { POPULAÇÃO ATENDIDA } \\
(\%)\end{array}$ & $\begin{array}{l}\text { POPULAÇÃO ATENDIDA } \\
\text { (hab.) }\end{array}$ & $\begin{array}{l}\text { PRODUZIDO } \\
\left(\mathrm{m}^{3} / \text { ano }\right)\end{array}$ \\
\hline 2007 & 1.822 .730 & 88,675 & 1.616 .309 & 129.786 .759 \\
\hline 2008 & 1.867 .503 & 88,729 & 1.657 .021 & 133.048 .843 \\
\hline 2009 & 1.913 .376 & 88,783 & 1.698 .758 & 136.392 .917 \\
\hline 2010 & 1.960 .375 & 88,837 & 1.741 .546 & 139.821 .041 \\
\hline 2011 & 2.008 .530 & 88,891 & 1.785 .412 & 143.335 .329 \\
\hline 2012 & 2.057 .867 & 88,946 & 1.830 .382 & 146.937 .945 \\
\hline 2013 & 2.108 .415 & 89,000 & 1.876 .486 & 150.631 .110 \\
\hline 2014 & 2.160 .206 & 89,054 & 1.923 .751 & 154.417 .100 \\
\hline 2015 & 2.213.269 & 89,108 & 1.972 .206 & 158.298 .247 \\
\hline 2016 & 2.267 .635 & 89,163 & 2.021 .881 & 162.276 .944 \\
\hline 2017 & 2.323 .336 & 89,217 & 2.072 .808 & 166.355 .642 \\
\hline 2018 & 2.380 .406 & 89,271 & 2.125 .018 & 170.536 .855 \\
\hline 2019 & 2.438 .878 & 89,326 & 2.178 .543 & 174.823 .160 \\
\hline 2020 & 2.498 .786 & 89,380 & 2.233 .416 & 179.217.197 \\
\hline 2021 & 2.560 .165 & 89,435 & 2.289 .671 & 183.721 .674 \\
\hline 2022 & 2.623 .052 & 89,489 & 2.347 .343 & 188.339.368 \\
\hline 2023 & 2.687 .484 & 89,543 & 2.406 .467 & 193.073 .124 \\
\hline 2024 & 2.753 .499 & 89,598 & 2.467 .081 & 197.925 .859 \\
\hline 2025 & 2.821 .135 & 89,653 & 2.529.221 & 202.900 .564 \\
\hline
\end{tabular}

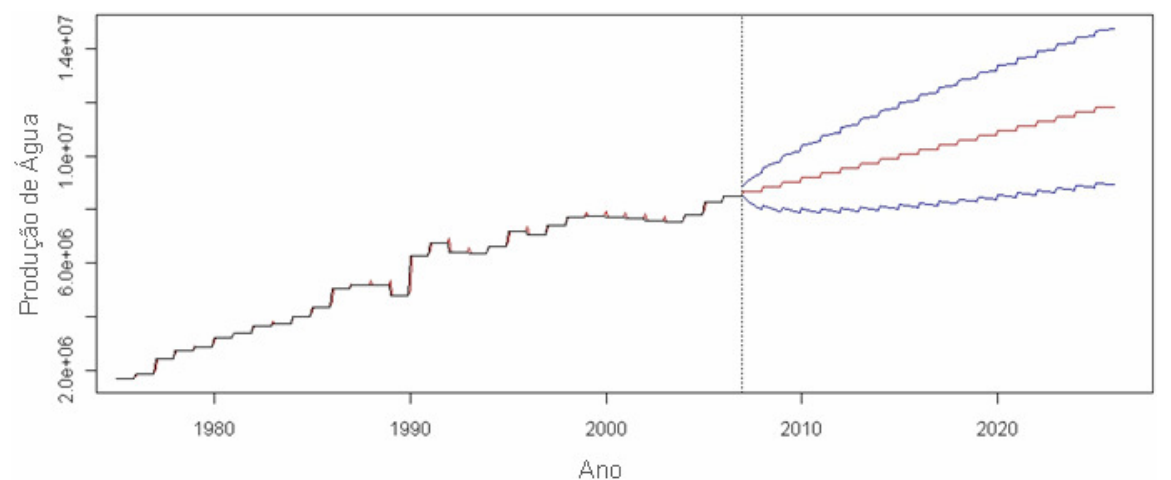

Figura 4. Produção de água da barragem do João Leite nos anos de 1975 a 2006, quantidade prevista de produção para os anos de 2007 a 2025 via método aditivo de Holt-Winters. 
Rev. Elet. em Gestão, Educação e Tecnologia Ambiental (e-ISSN: 2236-1170)

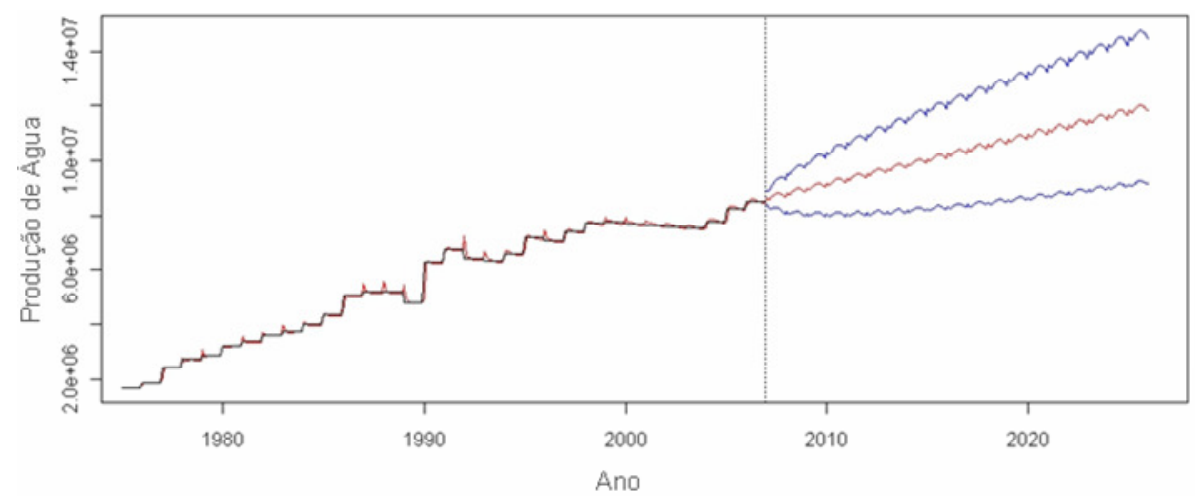

Figura 5. Produção de água da barragem do João Leite nos anos de 1975 a 2006, quantidade prevista de produção para os anos de 2007 a 2025 via método multiplicativo de Holt-Winters.

A estatística $U$ de Theil considera o custo desproporcional dos grandes erros e proporciona uma base relativa para comparações com métodos mais simples. Quanto mais próximo do zero for a estatística $U$ de Theil, melhor será a técnica de previsão usada. Valores iguais ou superiores a 1 (um) indicam que não há porque usar a técnica aplicada, pois qualquer método simples daria melhores resultados (MAKRIDAKIS, WHEELWRIGHT e HYNDMAN, 1998). Devido a isso o comportamento padrão da produção de água na Grande Goiânia da Barragem do João Leite parece melhor explicado por uma sazonalidade multiplicativa.

\section{CONCLUSÕES}

Devido a crescente demanda de água requerida para satisfazer a necessidade da população da Grande Goiânia associado com o impacto ambiental causado na criação de infraestrutura como as barragens e a pouca disponibilidade de água na natureza é que se fez necessário este estudo.

Uma previsão de qualidade para valores futuros de consumo de água pode-se melhorar, em muito, procedimentos intrínsecos ao processo global de distribuição de água, além, é claro, de corrigir possíveis problemas preexistentes de infraestrutura. Sendo assim, é de suma importância produzir uma boa previsão para a variável "água", a qual depende exclusivamente do usuário e de fatores que interferem no cotidiano do mesmo, como clima, a economia e o nível social.

Com o conhecimento destas análises foi possível auxiliar o planejamento de abastecimento de água tratada na região como também fornecer ferramentas estatísticas na detecção e solução de modelos de séries temporais.

Constatou-se nas previsões que entre 2023 e 2024 a Barragem do João Leite suportará o consumo de água da Grande Goiânia. Como a barragem atual tem capacidade para 196.762.243 $\mathrm{m}^{3}$ /ano, constatou-se nas previsões que entre 2023 e 2024 a Barragem do João Leite chegará a sua produção máxima e ainda conseguirá suportar todo o consumo de água da Grande Goiânia. Vale ressaltar que a Barragem do João Leite tem que estar sempre com sua capacidade total para que se possa garantir a previsão.

Todo este estudo só terá validade se ele puder conscientizar a população em relação ao uso da água, pois como podemos perceber, em um futuro não muito distante, teremos sim falta 


\section{Rev. Elet. em Gestão, Educação e Tecnologia Ambiental (e-ISSN: 2236-1170)}

de água mesmo com as duas principais represas da Grande Goiânia, Barragem do João Leite e Rio Meia Ponte tiverem em seu pleno funcionamento.

Se não forem adotadas ações preventivas, entre 2020 e 2030 teremos de dispor de fontes hídricas mais distantes da grande Goiânia, como por exemplo, a do Rio Caldas, causando impactos ambientais e de infraestrutura na região. Além disso, se as taxas de crescimento populacional mantiverem o ritmo de ascensão atual, por volta de 2024, os atuais mananciais de abastecimento da grande Goiânia estará no seu limite de utilização e assim deverá ser implementadas medidas mitigadoras para conter o avanço do desgaste com os mananciais.

\section{REFERÊNCIAS}

BOX, G. E. P.; JENKINS, G. Time Series Analysis: Forecasting and Control. Oakland: Holden-Day, 1976.

EHLERS, R.S. Análise de Séries Temporais. [S.L]: USP, 2009. Disponível em: <http://www.icmc.usp.br/ ehlers/stemp/stemp.pdf>. Acesso em 15 jan.2012.

HYNDMAN, R.J. \& KHANDAKAR, Y. "Automatic Time Series Forecasting: The forecast Package for R". Journal of Statistical Software, v. 27, ed. 3, Jul, 2008.

MAKRIDAKIS, S. G.; WHEELWRIGHT, S. C.; HYNDMAN, R. J. Forecasting: methods and applications. 3. ed. New York: John Willey \& Sons, 1998. 642 p.

MORETTIN, P. A.; TOLOI, C. M. C. Análise de Séries Temporais. São Paulo: Edgard Blucher, 2004. 537 p.

PELLEGRINI, F. R.; FLOGLIATTO, F. Estudo Comparativo entre modelos de Winters e de Box-Jenkins para a previsão de demanda sazonal. Revista Produto e Produção, v. 4, p. 72-85, Abril 2000.

RABELO, C. G. et al. Influência do uso do solo na qualidade da água no bioma Cerrado: um estudo comparativo entre bacias hidrográficas no Estado de Goiás, Brasil. Ambiente \& Água - An Interdisciplinary Journal of Applied Science, Taubaté, 4, 28 Julho 2009. 172-187.

REDE DAS ÁGUAS. Importância para a vida e distribuição no planeta. Rede das Águas, 2001. Disponível em: <http://www.rededasaguas.org.br/quest/quest_02.asp>. Acesso em: 25 Maio 2010.

SAMOHYL, R. W.; RUBSON, R.; MATTOS, V. L. D. Utilização do Método de Holt-Winters para Previsão do Leite Entregue às Indústrias Catarinenses. XX Encontro Nacional de Engenharia de Produção. Salvador: [s.n.]. 2001.

SANEAGO. Barragem do Ribeirão João Leite. Saneamento de Goiás S/A, 2010. Disponível em: <http://www.saneago.com.br/>. Acesso em: 20 Maio 2010.

SEPIN. Contagem da População. Superintendência de Estatística, Pesquisa e Informação, 2007. Disponível em: <http://www.seplan.go.gov.br/sepin/>. Acesso em: 08 Março 2010.

VIEIRA, A. D. R. Cadernos de Educação Ambiental Água para a Vida, Água para Todos: Livro das Águas. Brasília: WWF-Brasil, 2006. 72p. ISBN 85-86440-18-3. 